\title{
Comparação das condições de operação em regime permanente de geradores eólicos dos tipos i, ii e iv para análise em sistemas de distribuição com modelagem trifásica
}

\author{
Adriano Aron Freitas de Moura ${ }^{[1]}$, Francisco Clebson Sousa Galdino ${ }^{[2]}$, Ailson Pereira de Moura ${ }^{[3]}$, \\ Ednardo Pereira da Rocha ${ }^{[4]}$ \\ [1] Universidade Federal Rural do Semi-árido; adrianoaron@ufersa.edu.br \\ [2] Universidade Federal Rural do Semi-árido; \\ ${ }^{[3]}$ Universidade Federal do Ceará; ailson@ dee.ufc.br \\ ${ }^{[4]}$ Universidade Federal Rural do Semi-árido; ednardo.pereira@ufersa.edu.br \\ Recebido: 03/06/2019; \\ Aceito: 02/08/2019; \\ Publicado: 07/10/2019.
}

Resumo: Nos sistemas de distribuição de energia elétrica ocorrem as maiores causas dos desequilíbrios de tensão, principalmente devido à distribuição irregular de cargas por fase e ao emprego excessivo de transformadores monofásicos. Variações nas cargas monofásicas fazem com que as correntes nos condutores das três fases sejam diferentes, o que resulta em quedas de tensões diferentes, provocando desequilíbrio. $\mathrm{O}$ estudo de desequilíbrios de tensão na distribuição de energia é muito importante, uma vez que o bom funcionamento de equipamentos está diretamente relacionado a ele. No entanto, quando se trata da conexão de turbinas eólicas nos sistemas de distribuição, não se tem conhecimento de trabalhos acadêmicos que realizam um paralelo das condições de operação dos diversos componentes com a interconexão de diferentes configurações de geradores eólicos. O objetivo deste trabalho é analisar o problema de desequilíbrio de tensão em sistemas de distribuição mediante diversas situações de operação, principalmente com a inserção de turbinas eólicas tomando como base o sistema de 13 barras do IEEE. Além disso, são propostas e simuladas algumas maneiras de reduzir os desequilíbrios através do uso de técnicas de engenharia, como promovendo o balanceamento de cargas do sistema, conectando parques eólicos ao alimentador de distribuição, instalando bancos de reguladores de tensão monofásicos em subestações e corrigindo o fator de potência dos geradores das turbinas. Este trabalho mostrou que a modelagem de cargas, suas conexões, assim como a escolha do tipo de gerador (I, II e IV), podem influenciar significativamente no desequilíbrio de um sistema de distribuição. Além do mais, conclui-se que as técnicas propostas para reduzir o desequilíbrio da rede se mostraram eficazes e influenciaram também na redução das perdas globais do sistema e na melhoria dos níveis de carregamento e perfis de tensões.

Palavras-chave: fluxo de carga trifásico; sistemas de distribuição; geradores eólio-elétricos

\section{INTRODUÇÃO}

A interligação dos parques eólicos à rede elétrica das concessionárias de energia traz consigo algumas preocupações técnicas e econômicas. Para o estudo em questão a preocupação se restringe a análise do comportamento do sistema de distribuição de acordo com a tecnologia de geração a ser adotada, os critérios relacionados com a margem ou variação de queda de tensão, os carregamentos da linha e a controlabilidade gerada pelas diversas configurações de sistemas de energia eólica existentes, para integração nas redes elétricas. Neste artigo, são consideradas três configurações de sistemas de energia eólica operando com geradores de indução trifásicos, síncronos e por último o gerador de indução com resistência de rotor controlada. Vale ressaltar que para os três casos as máquinas estão acopladas diretamente na rede. Atualmente os geradores 
assíncronos (de indução trifásica), têm despontado como boa alternativa para aplicações em sistemas de energia eólica. Neste artigo é feito então um comparativo das duas tecnologias apresentadas no que diz respeito às suas vantagens e desvantagens para o sistema elétrico.

Alguns artigos relacionados com o tema são: Divnya K. and Rao P. S., que apresenta modelos de vários tipos de unidades geradoras de turbinas eólicas usadas como fontes de geração distribuída e demonstram suas aplicações para análise de estado estacionário [2]; e Moura, A. A. F. e Moura A. P., que demonstram o impacto das turbinas eólicas conectadas nos sistemas de distribuição e seus efeitos no equilíbrio da rede trifásica [3].

\subsection{Acoplamento de turbinas eólicas no sistema elétrico}

As máquinas eólicas que são alvo deste artigo (tipo I, II e IV), dependendo do tipo, podem ser acopladas ao sistema elétrico da maneira como mostrado na Figura 1 [1]:

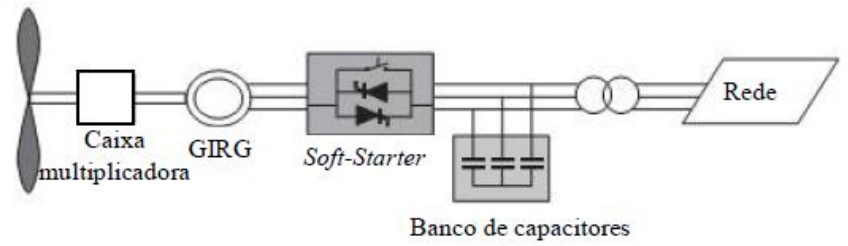

(a)

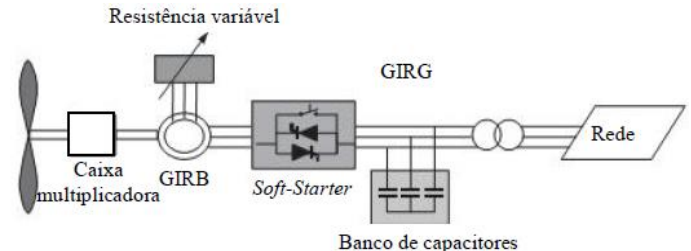

(b)

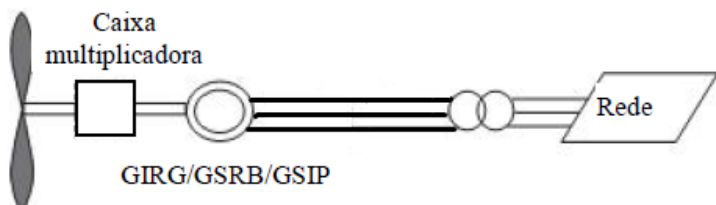

(c)

FiguRA 1. (a) Gerador de indução com rotor em gaiola (Tipo I); (b) gerador de indução com rotor bobinado e resistência de rotor controlada (Tipo II); (c) gerador síncrono diretamente conectado no sistema elétrico (Tipo IV).

A máquina do tipo I trata-se de um aerogerador operando com velocidade fixa e uma caixa multiplicadora. Um gerador de indução de rotor gaiola de esquilo (GIRG) é conectado diretamente à rede por meio de um transformador, como mostrado na Figura 1. O GIRG naturalmente absorve potência reativa da rede, por isso é utilizado um banco de capacitores para compensação da potência reativa e um Soft-Starter é usado para suavizar a conexão com a rede [1].

A turbina eólica do tipo II corresponde à turbina eólica de velocidade variável limitada com resistência de rotor variável. Como pode ser observado na Figura 1, o gerador de indução de rotor bobinado (GIRB) está conectado diretamente à rede elétrica. Assim como o tipo I, a máquina do tipo II também utiliza o banco de capacitores e o Soft-Starter pelos mesmos motivos citados acima. A diferença existente entre o gerador do tipo I e do tipo II é que o último possui uma resistência variável adicional ao rotor, que pode ser modificada por meio de um conversor controlado por um sensor óptico, fixado no eixo do rotor, sendo assim a resistência total do rotor é controlável, proporcionando o controle do escorregamento do gerador, que por consequência permite o controle da potência de saída do sistema.

Por fim o gerador eólico tipo IV mostrado na Figura 1, diz respeito à uma máquina eólica com ampla variação de velocidade (para o caso foi a máquina síncrona de rotor bobinado - GSRB). Para fins de simulação, o gerador não foi conectado por meio do conversor de frequência, sendo então o mesmo conectado diretamente na rede. Esse tipo também pode usar o gerador assíncrono de rotor bobinado (GIRB) e o síncrono com imã permanente (GSIP) [1]. As máquinas eólicas do tipo IV são responsáveis pela geração da maior parte da energia elétrica produzida no mundo. Uma vantagem das máquinas síncronas são o alto rendimento e o elevado fator de potência, o que as tornam economicamente viáveis e, além do mais, capazes de poder operar regulando a tensão como uma barra PV. 


\subsection{Modelagem do gerador de indução trifásico}

A modelagem matemática do gerador síncrono é baseada no circuito equivalente de Thevénin, tomando como base uma tensão interna e uma reatância própria da máquina. Será demonstrada a partir de agora a modelagem do gerador de indução, que serve de suporte para o entendimento da forma de operação dos aerogeradores que utilizam as máquinas do tipo I e II.

As correntes de fase da máquina são determinadas pela conversão das correntes de linha. Já as condições de operação interna são definidas pela análise completa da rede em que a máquina está ligada. O circuito equivalente monofásico da máquina de indução trifásica conectada em estrela está mostrado na Figura 2. No circuito da Figura 2 é aplicada a análise para as sequências positiva e negativa da rede elétrica. A componente de sequência zero é nula para esse tipo de máquina, pois, geralmente, sua conexão é feita em delta ou estrela isolada. A diferença entre as duas sequências está apenas entre o valor da resistência de carga RL, dada por:

$$
\mathrm{RL}_{\mathrm{i}}=\left(\frac{1-\mathrm{s}_{\mathrm{i}}}{\mathrm{s}_{\mathrm{i}}}\right) \times \mathrm{Rr}_{\mathrm{i}}
$$

Em que:

$\mathrm{i}=1$ para sequência positiva;

$\mathrm{i}=2$ para sequência negativa.

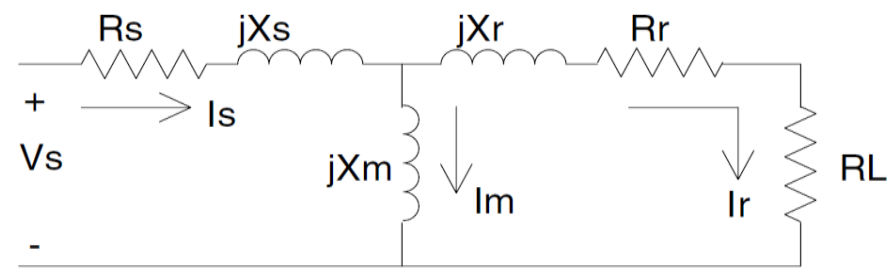

FIGURA 2. Circuito equivalente da máquina de indução [6].

O escorregamento na sequência positiva é dado por:

$$
\mathrm{s}_{1}=\frac{\mathrm{n}_{\mathrm{s}}-\mathrm{n}}{\mathrm{n}_{\mathrm{s}}}
$$

O escorregamento na sequência negativa é dado por:

$$
\mathrm{s}_{2}=2-\mathrm{s}_{1}
$$

As correntes de sequência negativas provocam a rotação da máquina no sentido inverso, produzindo uma perda de potência adicional e aquecimento do gerador. De acordo com [4], o gerador deve manter uma variação de tensão de no máximo $1 \%$. Se o valor do escorregamento de sequência positiva ( $\left.\mathrm{s}_{1}\right)$ é conhecido, então a sequência de impedância de entrada para as sequencias positivas e negativas da rede podem ser determinadas por:

$$
\mathrm{ZM}_{\mathrm{i}}=\mathrm{Rs}_{\mathrm{i}}+\mathrm{jXs_{i }}+\frac{\left(\mathrm{jXm} \mathrm{m}_{\mathrm{i}}\right)\left(\mathrm{Rr}_{\mathrm{i}}+\mathrm{RL}_{\mathrm{i}}+\mathrm{jXr} \mathrm{r}_{\mathrm{i}}\right)}{\mathrm{Rr}_{\mathrm{i}}+\mathrm{RL}_{\mathrm{i}}+\mathrm{j}\left(\mathrm{Xm}_{\mathrm{i}}+\mathrm{Xr}_{\mathrm{i}}\right)}
$$

As impedâncias de sequência na entrada são convertidas em admitâncias de entrada, sendo, portanto, o inverso das impedâncias. Logo, as correntes de sequência do gerador são:

$$
\mathrm{IM}_{\mathrm{i}}=\mathrm{YM}_{\mathrm{i}} \times \mathrm{VLN}_{\mathrm{i}}
$$

A conexão estrela da máquina de indução não possui neutro aterrado, de modo que sua corrente de sequência zero é nula. A tensão fase-neutro, por consequência, também será zero. A Equação 5 pode ser escrita na forma de matriz como:

$$
\left[\begin{array}{l}
\mathrm{IM}_{0} \\
\mathrm{IM}_{1} \\
\mathrm{IM}_{2}
\end{array}\right]=\left[\begin{array}{ccc}
1 & 0 & 0 \\
0 & \mathrm{YM}_{1} & 0 \\
0 & 0 & \mathrm{YM}_{1}
\end{array}\right]\left[\begin{array}{l}
\mathrm{VLN}_{0} \\
\mathrm{VLN}_{1} \\
\mathrm{VLN}_{2}
\end{array}\right] \Rightarrow\left[\mathrm{IM}_{012}\right]=\left[\mathrm{YM}_{012}\right]\left[\mathrm{VLN}_{012}\right]
$$

Pode-se obter a relação entre as correntes de fase e as tensões de fase nos terminais do gerador. Fazendo, então, a transformação de componentes simétricas para componentes de fase, tem-se: 


$$
\left[\mathrm{IM}_{\mathrm{abc}}\right]=\left[\mathrm{A}_{\mathrm{s}}\right]\left[\mathrm{IM}_{012}\right]
$$

Onde:

$$
\left[\mathrm{A}_{\mathrm{s}}\right]=\left[\begin{array}{ccc}
1 & 1 & 1 \\
1 & \mathrm{a}_{\mathrm{s}}{ }^{2} & \mathrm{a}_{\mathrm{s}} \\
1 & \mathrm{a}_{\mathrm{s}} & \mathrm{a}_{\mathrm{s}}{ }^{2}
\end{array}\right] \text { onde } \mathrm{a}_{\mathrm{s}}=1 \angle 120^{\circ}
$$

Por fim, de posse dessas equações, pode-se simular o comportamento das máquinas tipo I e II no sistema elétrico de potência, utilizando-se como base as correntes de fases obtidas do equacionamento acima. Um gerador de 500 HP (373 kVA) foi usado para obtenção dos principais parâmetros do sistema, tais como graus de desequilíbrios e perdas totais em termos de potência ativa e reativa do sistema de distribuição.

\section{MATERIAIS E MÉTODOS}

A metodologia aplicada neste artigo baseia-se na inserção de geradores eólio-elétricos do tipo I, II e IV no sistema de distribuição do IEEE, mas especificamente o modelo contendo 13 barras. Todos os geradores foram simulados conectados diretamente na rede elétrica, com a conexão das turbinas na barra 680 do sistema IEEE de 13 barras (Figura 3), composta de uma rede de distribuição radial, com um regulador de tensão na fonte, de um transformador entre as barras 633 e 634 e de uma chave seccionadora entre as barras 671 e 692 . Todas as características como carregamento das linhas, perfis de tensão em cada barra, grau de desequilíbrio, perdas elétricas, entre outros parâmetros estão descritos no documento fornecido pelo IEEE, denominado IEEE 13 Test Feeders, sendo portanto, dados que foram usados como referência para o sistema em seu caso base, para que possam ser confrontados com os valores resultantes das simulações com os geradores.

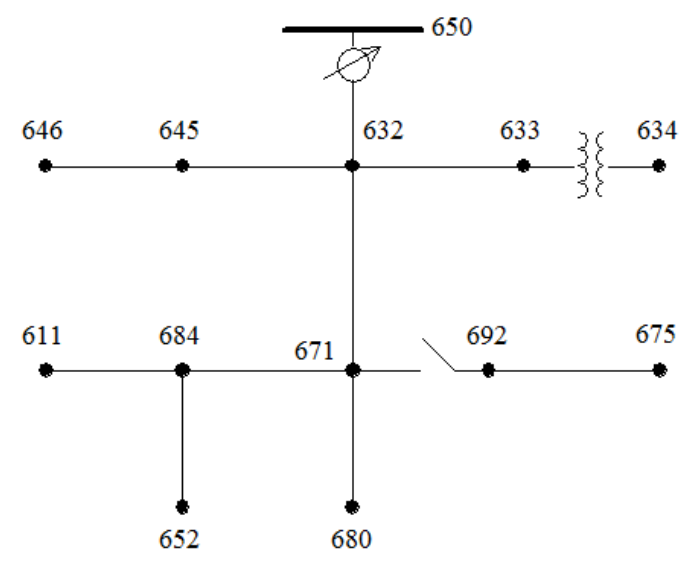

FIGURA 3. Sistema IEEE de 13 barras.

Sabendo que o escorregamento de um gerador deve ser negativo e não pode ser inferior a $-2 \%$, foi elaborado um programa no MATLAB com o intuito de encontrar um valor de escorregamento que levasse o gerador a operar com condições próximas de suas correntes nominais nas três fases, para tal foi usada a análise da máquina de indução presente em [4] quando a mesma está sujeita a tensões desequilibradas e se recorre ao método das componentes simétricas. Uma vez que o valor do escorregamento de sequência positiva $\left(\mathrm{s}_{1}\right)$ é conhecido, então as impedâncias de entrada para as sequências positivas e negativas da rede podem ser determinadas e por fim, dividindo-se a tensão fase-neutro de sequência pela sua respectiva impedância encontram-se os valores de correntes. Após sucessivos testes do programa em questão obteve-se um escorregamento ótimo de $-1,3 \%$, que produziu nas três fases as seguintes correntes de linha (em modulo): Ia = 49,50 A, Ib $=51,86 \mathrm{~A}$ e Ic = 58,75 A, o fato das correntes possuírem valores diferentes nas três fases é normal uma vez que desde a sua origem o sistema estudado é desequilibrado, além do mais esses valores estão relativamente próximos da corrente nominal utilizando os dados de placa da máquina que indica uma corrente de aproximadamente $52 \mathrm{~A}$ (para uma máquina de $373 \mathrm{kVA}$ ) em condições equilibradas de operação.

A fim de fornecer condições iguais para analisar o comportamento do gerador de indução e do gerador síncrono (que possui fator de potência de 0,98), foi necessária proceder com a correção do primeiro para um fator de potência de também de 0,98 (tanto capacitivo quanto indutivo) por meio da inserção de um banco de 
capacitores trifásicos na barra 680 do sistema. Para tanto foi elaborado um outro programa no MATLAB. Para encontrar o banco de capacitores inicialmente foi usado os valores de tensões fase-neutro e de correntes do caso base em cada fase para calcular a potência aparente do gerador e poder obter os fatores de potência antes da correção, que foram FPa de 0,9391, FPb de 0,8701 e FPc de 0,9158. Os valores numéricos dos bancos de reativos em $\mathrm{kVAr}$ encontrados foram de 18,85, 36,85 e 31,45 kVAr nas fases A, B e C respectivamente, os valores diferentes em cada fase do banco se deve ao fato de que o sistema naturalmente já é desequilibrado. Esse banco é para um FP = 0,98 indutivo. De forma semelhante foi encontrado um banco de capacitores em kVAr para um FP = 0,98 capacitivo: 64,05, 79,31 e 85,12 nas fases A, B e C respectivamente. De posse desses dados foram realizadas as simulações descritas no próximo tópico.

\section{RESULTADOS E DISCUSSÕES}

Tomando-se como referência o caso base do sistema IEEE 13 barras, foram calculadas as perdas elétricas quando está conectado o gerador de indução e o gerador síncrono, como mostrado nas Tabelas 1 e 2 abaixo:

TABela 1. Total de Perdas $-\mathrm{FP}=0,98$ indutivo X FP = 0,98 capacitivo - Gerador de Indução de 500HP.

\begin{tabular}{lcccccc}
\hline & $\begin{array}{c}\text { FASE A } \\
(k W)\end{array}$ & $\begin{array}{c}\text { FASE A } \\
(k V A r)\end{array}$ & $\begin{array}{c}\text { FASE B } \\
(k W)\end{array}$ & $\begin{array}{c}\text { FASE B } \\
(k V A r)\end{array}$ & $\begin{array}{c}\text { FASE C } \\
(k W)\end{array}$ & $\begin{array}{c}\text { FASE C } \\
(k V A r)\end{array}$ \\
\hline $\mathrm{FP}=0,98 \mathrm{IND}$ & 32,548 & 133,661 & $-2,889$ & 32,847 & 66,534 & 109,114 \\
\hline $\mathrm{FP}=0,98 \mathrm{CAP}$ & 32,506 & 129,351 & $-3,408$ & 32,680 & 63,813 & 103,478 \\
\hline
\end{tabular}

${ }^{1}$ a - Perdas totais (Fator de potência indutivo): 96,194 kW e 275,623 kVAr; b - Perdas totais (Fator de potência capacitivo): $92,910 \mathrm{~kW}$ e $265,509 \mathrm{kVAr}$.

TABela 2. Total de Perdas $-\mathrm{FP}=0,98$ indutivo $\mathrm{X} F \mathrm{FP}=0,98$ capacitivo - Gerador síncrono de 500HP ligado diretamente na rede.

\begin{tabular}{lcccccc}
\hline & $\begin{array}{c}\text { FASE } \\
(k W)\end{array}$ & $\begin{array}{c}\text { FASE } \\
(k V A r)\end{array}$ & $\begin{array}{c}\text { FASE B } \\
(k W)\end{array}$ & $\begin{array}{c}\text { FASE B } \\
(k V A r)\end{array}$ & $\begin{array}{c}\text { FASE C } \\
(k W)\end{array}$ & $\begin{array}{c}\text { FASE C } \\
(k V A r)\end{array}$ \\
\hline $\mathrm{FP}=0,98 \mathrm{IND}$ & 31,655 & 141,738 & $-5,257$ & 29,098 & 71,466 & 110,337 \\
\hline $\mathrm{FP}=0,98 \mathrm{CAP}$ & 31,041 & 137,904 & $-6,129$ & 29,209 & 70,467 & 106,632 \\
\hline
\end{tabular}

${ }^{1}$ a - Perdas totais (Fator de potência indutivo): 97,864 kW e 281,172 kVAr; b - Perdas totais (Fator de potência capacitivo): $95,379 \mathrm{~kW}$ e $273,745 \mathrm{kVAr}$.

Por análise das Tabelas, percebe-se que o gerador de indução apresenta menores perdas elétricas tanto de potência ativa quanto de potência reativa, se comparado com o gerador síncrono, quando inserido no sistema, sendo que o fator de potência capacitivo da máquina de indução apresentou o melhor desempenho.

TaBela 3. Graus de Desequilíbrios de Tensão do Gerador de Indução X Gerador Síncrono - Caso Base.

\begin{tabular}{|c|c|c|}
\hline Barra 680 & GD $(\%)$ - Gerador de indução & GD (\%) - Gerador síncrono \\
\hline $\mathrm{Fp}=0,98$ Indutivo & 1,5570 & 1,7817 \\
\hline $\mathrm{Fp}=0,98$ Capacitivo & 1,5773 & 1,8059 \\
\hline
\end{tabular}

Os valores de referência para o grau de desequilíbrio sem a presença das turbinas eólicas são de 1,9187\% para o período de carga pesada e de $0,6804 \%$ para o período de carga leve. Analisando-se a Tabela 3, nota-se que ao se inserir no sistema de distribuição, para o regime de cargas pesadas, o gerador de indução possui um menor grau de desequilíbrio na barra 680 se comparado com o gerador síncrono, sendo 1,5570\% para operação com $\mathrm{Fp}$ de 0.98 indutivo e $1,5773 \%$ para o $\mathrm{Fp}=0,98$ capacitivo, vale ressaltar que de qualquer maneira a presença de uma turbina eólica no sistema (seja do tipo I, II ou IV) contribui para a diminuição do grau de desequilíbrio. Para obter resultados de simulações com o sistema de distribuição operando em regime de carga leve, todas as cargas do sistema (concentradas e distribuídas) do sistema 13 barras foram juntadas numa planilha e o valor de cada potência por fase, tanto a parte ativa $(\mathrm{kW})$ e reativa $(\mathrm{kVAr})$, foi dividido por três e o resultado obtido foi colocado no respectivo nó do sistema no lugar dos valores de cargas antigos. A máquina de indução apresentou resultados um pouco melhores, pois isso seus resultados são mostrados abaixo. Novamente foram calculados os graus de desequilíbrio nas barras e as perdas elétricas nas linhas. Vale ressaltar que o carregamento da linha onde foi inserido a turbina eólica pouco se alterou nos diversos modos de simulação. 
Tabela 4. Total de Perdas - Perdas Totais do Gerador de Indução para o Regime de Carga Leve.

\begin{tabular}{lcccccc}
\hline & $\begin{array}{c}\text { FASE } \\
(k W)\end{array}$ & $\begin{array}{c}\text { FASE } \\
(k V A r)\end{array}$ & $\begin{array}{c}\text { FASE B } \\
(k W)\end{array}$ & $\begin{array}{c}\text { FASE B } \\
(k V A r)\end{array}$ & $\begin{array}{c}\text { FASE C } \\
(k W)\end{array}$ & $\begin{array}{c}\text { FASE C } \\
(k V A r)\end{array}$ \\
\hline $\mathrm{FP}=0,98 \mathrm{IND}$ & 3,155 & 7,503 & 0,543 & 3,485 & 3,302 & 6,219 \\
\hline $\mathrm{FP}=0,98 \mathrm{CAP}$ & 3,289 & 7,029 & 0,674 & 4,258 & 3,290 & 6,865 \\
\hline
\end{tabular}

${ }^{1}$ a - Perdas totais (Fator de potência indutivo): 7,000 kW e 17,207 kVAr; b - Perdas totais (Fator de potência capacitivo): $7,254 \mathrm{~kW}$ e $18,152 \mathrm{kVAr}$.

Examinando-se a Tabela 4, percebe-se que para o regime de carga leve as perdas elétricas do sistema caem bruscamente se comparados com o caso base (com cargas pesadas), sofrendo uma redução de $89.194 \mathrm{~kW}$ e 258.416 kVAr, ambas para um fator de poência indutivo da máquina síncrona. Já a Tabela 5, mostra os graus de desequilibrios dos sistema para o regime de carga leve, também na barra 680:

TABEla 5. Graus de Desequilíbrios de Tensão do Gerador de Indução X Gerador Síncrono - Carga.

\begin{tabular}{|c|c|c|}
\hline Barra 680 & GD (\%) - Gerador de indução & GD (\%) - Gerador síncrono \\
\hline $\mathrm{Fp}=0,98$ Indutivo & 0,5653 & 0,6023 \\
\hline $\mathrm{Fp}=0,98$ Capacitivo & 0,6106 & 0,6432 \\
\hline
\end{tabular}

Percebe-se que, de acordo com a Tabela 5, os graus de desequilíbrios para todos os casos caiu para praticamente um terço dos valores originais, como por exemplo, ocorreu uma redução de 66,20\% no grau de desequilíbrio no gerador síncrono operando com fator de potência indutivo.

Com o intuito de estudar o sistema de distribuição operando com carga equilibrada, todas as cargas do sistema (concentradas e distribuídas) do sistema 13 barras foram balanceadas da seguinte forma: em cada nó foi somada as potências ativas (das três fases), o resultado foi dividido por três e colocado nas respectivas fases A, B e C (o mesmo valor foi utilizado nas três fases do nó), o mesmo procedimento foi adotado para as potências reativas presentes em cada nó do sistema. Novamente foram calculados os graus de desequilíbrio nas barras, as perdas elétricas, os perfis de tensões para o caso de cargas equilibradas. A Tabela 6 mostrada a seguir, um resumo dos graus de desequilibrios em porcentagem para as várias configurações testadas durante o trabalho, tais como operação do sistema sem regulador de tensão, equilibrio das cargas nos nós trifásicos e mudança de conexão das cargas (ligação em delta (D) ou estrela (Y)).

TABela 6. Graus de Desequilíbrios de Tensão do Gerador de Indução X Gerador Síncrono.

\begin{tabular}{ccccccc}
\hline Barra 680 & \multicolumn{3}{c}{ Gerador de indução } & \multicolumn{3}{c}{ Gerador síncrono } \\
\hline $\begin{array}{c}\text { Fator de } \\
\text { potência }\end{array}$ & $\begin{array}{c}\text { Operação sem } \\
\text { regulador de } \\
\text { tensão }\end{array}$ & $\begin{array}{c}\text { Equilibrio } \\
\text { das cargas }\end{array}$ & $\begin{array}{c}\text { Conexão } \\
\text { das cargas }\end{array}$ & $\begin{array}{c}\text { Operação sem } \\
\text { regulador de } \\
\text { tensão }\end{array}$ & $\begin{array}{c}\text { Equilibrio } \\
\text { das cargas }\end{array}$ & $\begin{array}{c}\text { Conexão } \\
\text { das cargas }\end{array}$ \\
\hline $\begin{array}{c}\mathrm{FP}=0,98 \\
\text { Indutivo }\end{array}$ & 2,3359 & 1,5614 & $\begin{array}{c}1,4847(\mathrm{Y}) \\
1,2224(\mathrm{D})\end{array}$ & 2,6945 & 1,7670 & $\begin{array}{c}1,6540(\mathrm{Y}) \\
1,1439(\mathrm{D})\end{array}$ \\
\hline $\begin{array}{c}\mathrm{FP}=0,98 \\
\text { Capacitivo }\end{array}$ & 2,3423 & 1,5432 & $\begin{array}{l}1,4812(\mathrm{Y}) \\
1,0443(\mathrm{D})\end{array}$ & 2,6972 & 1,7554 & $1,4380(\mathrm{Y})$ \\
$1,1547(\mathrm{D})$
\end{tabular}

Considerando a Tabela 6, percebe-se a importância do regulador para manter o nível de desequilibrio de tensão em valores aceitáveis, pois sem a presença do mesmo o grau de desequilibrio aumenta cerca de $41 \%$ na barra 680 para a operação do sistema com o gerador síncrono. Também nota-se o quanto é importante equilibrar as cargas nos nós do sistema a fim de diminuir o desequilibrio que caiu em torno de $20 \%$ também na barra 680 para a simulação com o gerador de indução. Por fim uma possível mudança na conexão das cargas do sistema, sendo todas conectadas em estrela ou todas em delta também se mostrou como uma alternativa eficaz para diminuir as variações do sistema, com destaque quando todas as cargas foram ligadas em delta em que o desequilibrio decresceu cerca de $46 \%$ para o gerador de indução operando com fator de potência 0,98 capacitivo.

O perfil das tensões na barra de conexão foi também anotado para diversos casos já citados (Figura 4). Segundo [5], o nível de tensão em regime permanente, deve permanecer na faixa de 0,93 à 1,05 em relação ao valor nominal de 1 p.u., para uma faixa de tensão superior $1 \mathrm{kV}$ e menor que $69 \mathrm{kV}$, o valor da tensão na barra 680 na fase B está, então, fora dos padrões estabelecidos por norma, uma vez que apresenta o valor em módulo de 1,0529 p.u nestas condições, para o regime original com carga pesada e sem aerogeradores. 


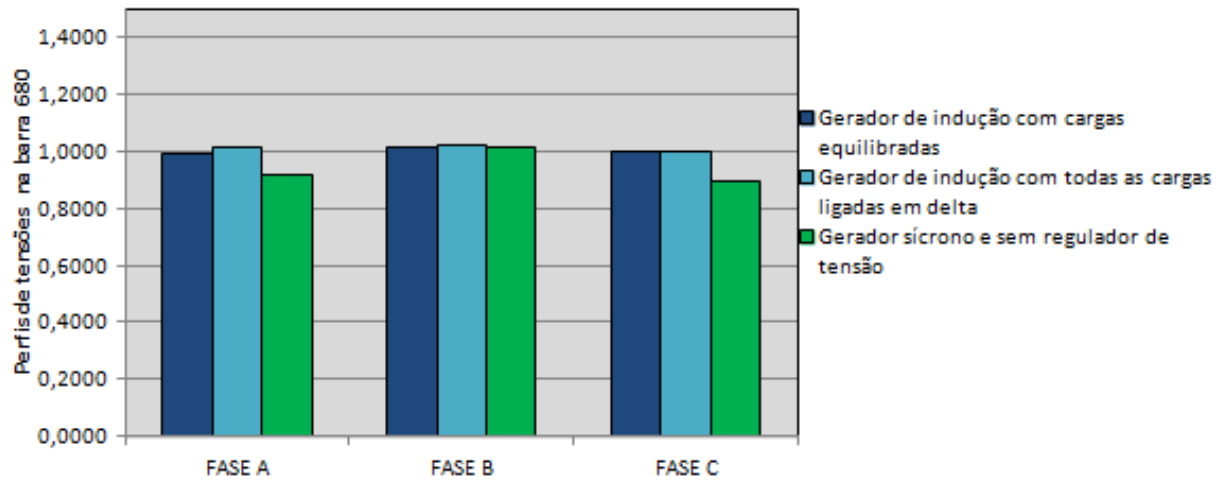

FIGURA 4. Principais perfis de tensões encontrados durante as simulações com as turbinas eólicas na barra 680.

De uma forma geral, todas as simulações testadas para o gerador de indução trifasico presente na barra 680 indicaram uma melhora nos perfis de tensões nas três fases, com as mesmas estando sempre nos níveis exigidos por norma. Porém, com a inserção do gerador síncrono presente na barra 680 os perfis de tensões pioraram em praticamente todas as formas de simulação citadas acima, se mantendo nos níveis aceitáveis apenas nos casos em que foi simulado com o regime de cargas equilibradas e com regime de cargas leves, o que evidencia a necessidade de um maior cuidado quando se deseja empregar esse tipo de máquina em um parque eólico. A Figura 4 mostra ainda os melhores resultados obtidos com as simulações para o gerador de indução no sistema com carga equilibrada e FP de 0,98 indutivo e com a máquina de indução em que todas as cargas do sistema estão ligadas em delta para um FP de 0,98 capacitivo, contrastando com o gerador síncrono com fator de potência 0,98 indutivo e sem a presença do regulador de tensão com valores fora da norma nas fases $\mathrm{B}$ e C na barra 680 .

Não se pode comparar diretamente as características de operação da máquina do tipo II com os demais geradores, devido ao fato de que a potência é alterada para cada valor de escorregamento, mudando então todos os parâmetros. Tomando-se como base um valor de resistência externa variando com intervalos de $0,1 \Omega$ partindo de $\mathrm{Rr}=0,7124 \Omega$ (valor de placa do gerador de $500 \mathrm{hp}$ ) e com um escorregamento flutuando de $-2 \%$ a $-4 \%$ com intervalos de $-0,5 \%$, analisando a Figura 5 percebe-se que o GIRB quando inserido na barra 680 diminui o grau de desequilibrio (caso base), principalmente para um fator de potência capacitivo.

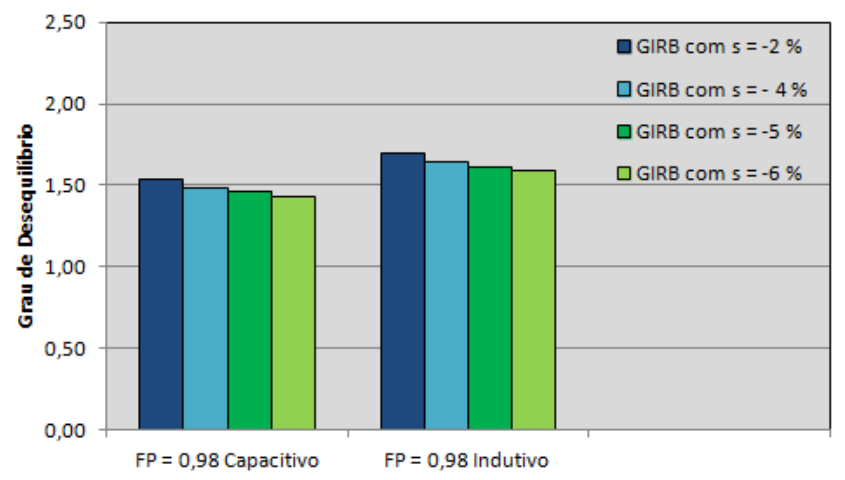

FIGURA 5. Graus de desequilíbrios com o GIRB conectado na barra 680.

\section{CONCLUSÃO}

Este artigo demonstrou que a modelagem de cargas, suas conexões, balanceamento das cargas e tipo de gerador utilizado podem influenciar significantemente no grau de desequilíbrio de uma sistema de distribuição de energia elétrica, nas perdas globais e nos perfis de tensões. Demonstrou-se também que o sistema IEEE 13 barras sofre maiores variações com a operação com carga pesada, especialmente quando é retirado o regulador de tensão do nó fonte (barra 651). O gerador de indução foi o que mais contribuiu para a diminuição das perdas (em kW e kVAr) e também foi o que controlou de forma mais satisfatória os graus de desequilibrios do sistema, principalmente para o regime de cargas leve. Das diversas configurações testadas a fim de melhorar os parêmetros de funcionamento do arranjo estudado, a conexão de todas as cargas (em estrela ou em delta) se 
mostrou uma alternativa bastante interessante para mitigar os desequilibrios de tensão, com destaque para as conexões das cargas em delta, que proporcionaram um redução de $45,55 \%$ (para o gerador de indução com fator de potência capacitivo) no desequilibrio na barra 680. Já quanto se trata de perfis de tensões, a máquina de indução também se mostrou mais eficaz que a síncrona para manter os níveis te tensões mais próximos de 1 p.u, além disso, fica evidente a importância do regulador de tensão para a boa operação do sistema de distribuição.

\section{AGRADECIMENTOS}

À UFERSA pela oportunidade de pesquisa na área de sistemas elétricos de potência.

\section{REFERÊNCIAS}

[1] ACKERMANN, Thomas. Wind Power in Power Systems. Sweden: Ed John Wiley and Sons, 2005.

[2] DIVNYA, K. C. and RAO, P. S. N., "Models for Wind Turbine Generating Systems and threir Application in Load Flow Studies", Electric Power Systems Research, vol. 76, pp. 844-856, 2006.

[3] MOURA, A. A. F. and MOURA, A. P. "Impact of connected wind turbines on steady-state operation of three-phase radial distribution network", in INDUSCON, 2008.

[4] KERSTING, W. H., Distribution System Modeling and Analysis, CRC press, 4nd Edition, New York, USA, 2017.

[5] ANEEL - Agência Nacional de Energia Elétrica, Resolução $N^{\circ}$ 505/2001 Disponível em: http://www.aneel.com.br. 